\title{
Polymer Nanocomposite Processing, Characterization and Applications 2011
}

\author{
Gaurav Mago, ${ }^{1}$ Dilhan M. Kalyon, ${ }^{2}$ and Sadhan C. Jana ${ }^{3}$ \\ ${ }^{1}$ Department of Mechanical Engineering, Stevens Institute of Technology, Hoboken, NJ 07030, USA \\ ${ }^{2}$ Highly Filled Materials Institute and Department of Chemical Engineering and Materials Science, Stevens Institute of Technology, \\ Hoboken, NJ 07030, USA \\ ${ }^{3}$ Department of Polymer Engineering, The University of Akron, 250 South Forge Street, Akron, OH 44325, USA \\ Correspondence should be addressed to Gaurav Mago, gauravmago2001@gmail.com
}

Received 14 December 2011; Accepted 14 December 2011

Copyright (c) 2011 Gaurav Mago et al. This is an open access article distributed under the Creative Commons Attribution License, which permits unrestricted use, distribution, and reproduction in any medium, provided the original work is properly cited.

There is a growing interest in the incorporation of nanoparticles into polymers to improve various functional properties (such as mechanical, thermal, optical, magnetic, and electrical properties). However, the ultimate properties of nanocomposites are affected by a large number of factors including the microstructural distributions that are generated during nanocomposite processing. While significant work has been done on preparation and properties of polymer nanocomposites, the interrelationship between processing, morphology, and functional properties of nanocomposites is complex and needs further elucidation. Furthermore, the effective utilization of nanoparticles in polymers depends strongly on the ability to disperse the nanoparticles uniformly throughout the polymeric matrices especially without reducing their aspect ratios. An understanding of the relationship between processing, morphology, and functional properties of nanocomposites will be very helpful in optimizing the overall properties of nanocomposites as well as improving the models for predicting the properties of nanocomposite systems. Such knowledge will facilitate the generation and optimization of polymeric nanocomposites with better tailored ultimate properties.

Considering the challenges in the area of development of adequate methods of processing for nanocomposites, we invited research articles to this special issue with a special focus on the interrelationships between polymer nanocomposite processing, characterization, and novel applications. This special issue with a total of seven papers covers a wide range of areas related to covalent functionalization of carbon nanotubes (CNTs) with polyurethane segments to improve the dispersion and mechanical properties; silanization of CNTs to improve interaction at the interface to allow better load transfer between matrix and nanoparticles; rheological characterization of nanoclay dispersions in nanocomposites prepared via different processing methods such as sonication, manual mixing, or high shear mixing; electrospinning to produce nanofibrous resonant membranes for acoustic applications; superhydrophobic membranes prepared via electrospinning from fluorinated silane-functionalized pullulan/poly(vinyl alcohol) blends; electrical properties of doped nematic liquid crystals; and finally, the preparation of polymer nanocomposites with graphite and nickel nanoparticles for potential applications in positive temperature coefficient of resistance devices, self-regulating heater, and temperature sensors.

It is hoped that this special issue will help readers with a wide range of backgrounds to understand the impact of various processing methods as well as nanoparticles on the nanocomposite properties and applications.

\section{Acknowledgments}

The editors would like to acknowledge the invited and contributing authors, reviewers, and staff members of Hindawi Publisher.

Gaurav Mago 
Dilhan M. Kalyon Sadhan C. Jana 

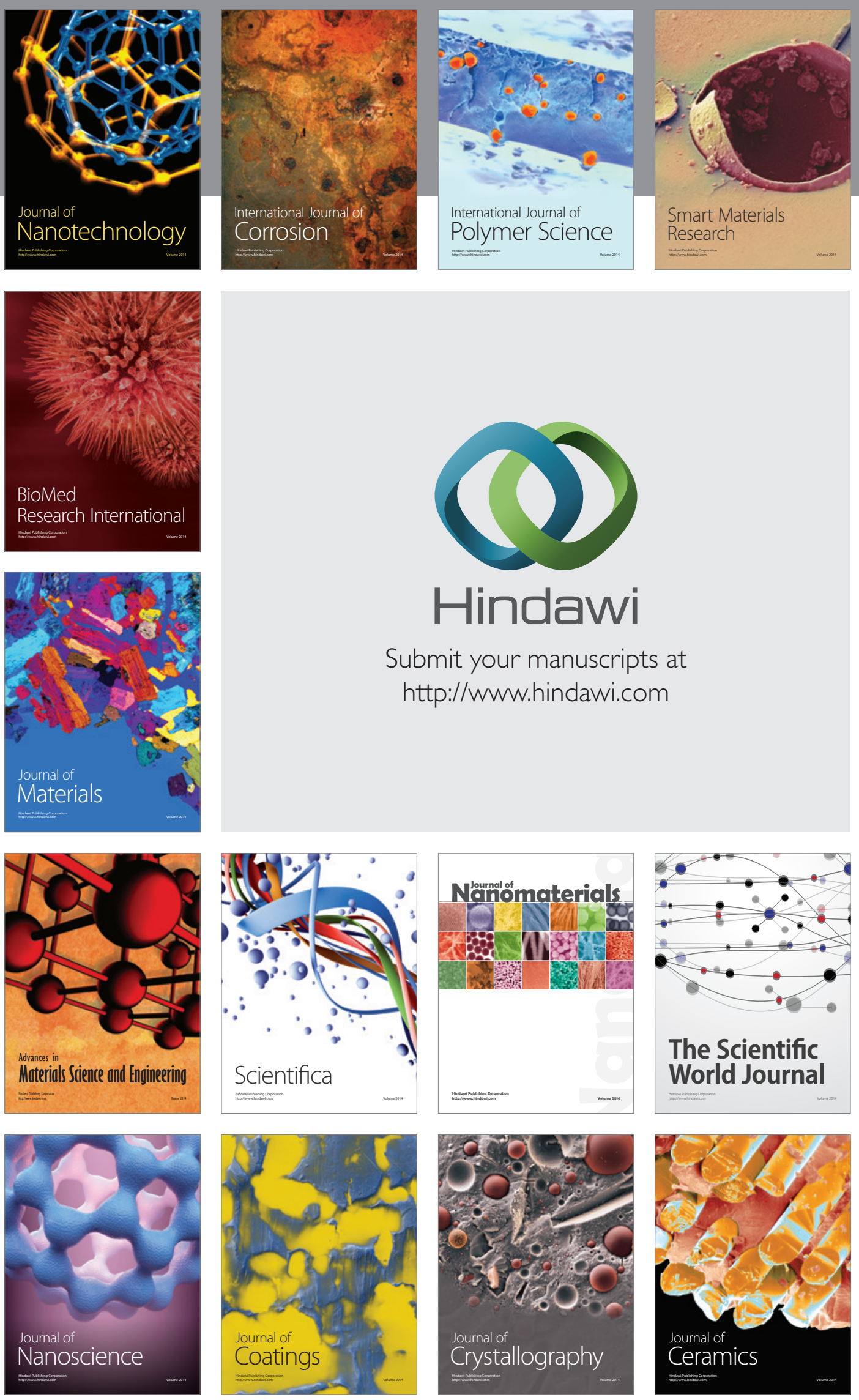

The Scientific World Journal

Submit your manuscripts at

http://www.hindawi.com

\section{World Journal}

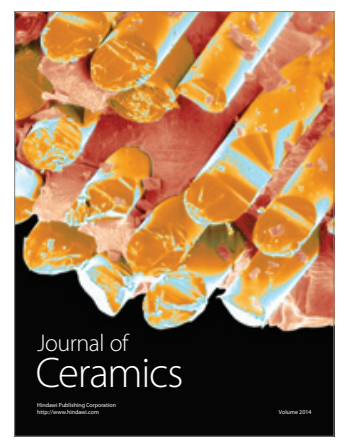

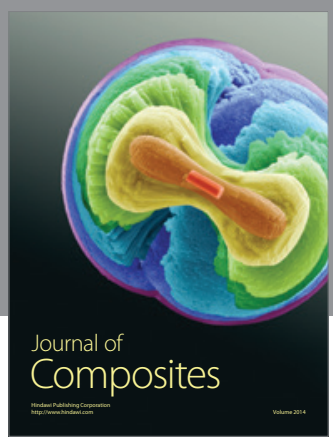
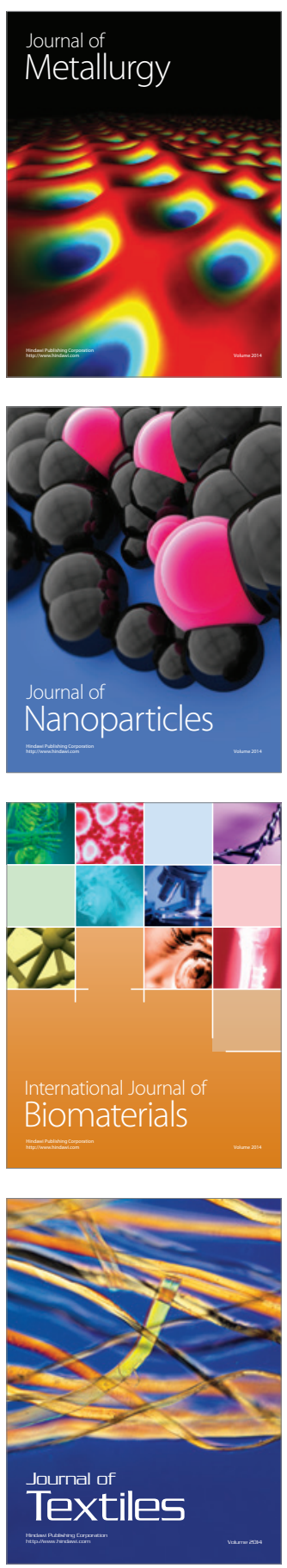